\title{
Health Care of the Deaf - Toward a New Understanding
}

\author{
Frank Hochman, MD
}

I have been asked to contribute this guest editorial to provide further perspective on the health care experience of elderly deaf patients reported in the article by Witte and Kuzel in this issue. ${ }^{1}$ Accordingly, this editorial explores the availability and problems of medical care for the elderly Deaf in Richmond, $\mathrm{Va}$, area as well as comments on health care delivery to the Deaf population at large. As in the accompanying article, I, too, will use the word deaf to represent people with severe hearing impairment and Deaf to represent the subset that feels linked culturally.

From my experiences as a graduate of a school for the Deaf (The New York School for the Deaf, popularly known as PS 47), as the first graduate of a Deaf school to earn his medical degree, and as the former physician for the California School for the Deaf and the California School for the Blind (Fremont, Calif), my comments might be quite upsetting and controversial to many Deaf and professionals in the field of the (d)Deaf.

As I see it and as the authors point out, the root of the problem of health care delivery to the Deaf is communication. I am not speaking of communication in the limited sense of physician and patient conversing, but in the broader context, such as, calling the physician's office, the physician's office calling the patient, or a deaf patient calling the laboratory, pharmacy, hospital, or specialist. Here the greater onus is on the Deaf person, who faces the challenge of good communication all his or her life. Medical professionals, on the other hand, have very limited exposure to and knowledge of the Deaf. Every (d)Deaf patient should inform his or

Submitted 8 December 1999.

From a private practice. Address reprint requests to Frank Hochman, MD, 1999 Mowry Ave, Suite L, Fremont, CA 94538.

Dr. Hochman is the founder of the Society of HearingImpaired Physicians (SHIP). Interested parties can contact him by e-mail at the following address: tphship@aol.com. her physician's office of the local relay service and telephone devices for the deaf ([TDDs] along with the special TDD language that facilitates calls on the TDD).

The authors write that participants seemed resigned to these circumstances (ie, various barriers to health care delivery). This attitude will solve nothing. The Deaf themselves should fight for better understanding of Deaf culture. Did the hearing world fight for "Deaf President Now" - when the students at Gallaudet University in Washington, DC, fought to have a Deaf person selected as president of their school?

I took the liberty of asking several of my Deaf patients to read the article and to give me their impressions. I did the same with my back-office assistant (who has been with me for more than 5 years and has worked with and helped many of my (d)Deaf patients).

My Deaf patients were surprised at some of the comments by other Deaf in the article. The comment about the staff being frightened and shaking in the presence of a Deaf person was thought to be absurd.

If one reads carefully and can forget momentarily that the group studied is Deaf, one will notice the great similarity between their complaints and those elicited when studying hearing persons:

"I can't get the doctor's office on the telephone."

"I can't get the laboratory results."

"The doctor uses big words that I can't understand."

"The doctor or nurses or office secretary speaks too quickly."

I want to address the question of physicians unprepared to meet the needs of deaf patients effectively and sensitively. Although these points are valid, it must be borne in mind that the average primary care physician will care for very few Deaf patients in a lifetime. To ask physicians to spend time studying for a problem that is not life-threat- 
ening will meet with little, if any, enthusiasm. Those practicing in areas with large Deaf populations, such as Washington, DC, or Rochester, NY, however, should endeavor to learn about the Deaf culture.

American Sign Language (ASL) might prove too difficult and time consuming for most physicians to learn well enough to understand many Deaf patients. One only has to look at how few hearing parents of deaf children learn ASL well enough to truly converse with their own children.

As for waiting in the reception room (never call it a waiting room) and not hearing their name called - my Deaf patients all tell me of the same problem at one time or another, and they all solve it simply by telling the receptionist, "I am deaf and will be sitting here," (but add) "I will not hear you calling me and YOU must make sure you get my attention."

As for what can be done now - the old suggestions found in many medical journals and magazines are still valid. Among them are the following:

1. Face the patient at all times.

2. KISS (Keep It Simple Stupid). No long words. No fast talking.

3. Repeat. If you think you are not being understood, you might see a blank look on the patient's face - just as you would on a hearing patient's face. Repeat, and ask the patient what you said.

4. Provide good lighting. Dark is okay for blind patients, not deaf patients.

5. If you have a beard or moustache, shave it.

Unlike reported in the article, I do not find that my Deaf patients make more visits than do their hearing counterparts. My own experiences, however, might be skewed because I speak ASL, and both my patients and I understand one another. As for the observation that the Deaf have more difficulties understanding physicians, it must be kept in mind that the average reading level and command of written English of the Deaf is considerably below that of the average hearing person. This regrettable, but accurate, fact poses a major problem in health care for the Deaf. Furthermore, the authors' comment that "the relay operator sometimes types the hearing person's voiced words too fast," highlights an additional problem. TDDs are inherently slow! Finally, many Deaf persons have not been taught such simple activities as going to the pharmacy, taking medicines as prescribed, or re- $\mathrm{J}$ turning to the physician for a follow-up visit.

Some items in the article are confusing. The authors mention focus groups. How were these $\frac{0}{3}$ groups selected? The age criteria of " 55 years or 0 older" should have been divided into "young old" (55 to 70 years), "old" (70 to 80 years) and "very $\underset{\overrightarrow{0}}{\overrightarrow{0}}$ old" ( 80 years and older). I have noticed that many of my very old Deaf patients have a habit of fingerspelling words that have an easy ASL sign. Those who are 55 to 60 years of age sign very much as do their younger (20-to 50-year-old) friends and family. Many offices use voice mail, which eliminates the possibility of a Deaf patient leaving messages (or "hearing" the instructions). This problem can be avoided by using the relay service that most telephone companies provide.

There does exist a certain amount of ignorance about the Deaf. Once during surgery I was discussing the need for an ASL interpreter for Deaf patients. The anesthesiologist expressed shock at the need for $\$ 60$ an hour for the interpreter, declaring that it cost her less for an interpreter for Chinese, Indian, or Vietnamese patients. I informed her that these people can learn to speak English. The Deaf, for the most part, cannot speak any language. I still have to correct my colleagues not to write or say "deaf and dumb" or "deaf-mute." Civility and civilizations take time.

The closing comments and recommendations by Witte and Kuzel are accurate and relevant; nevertheless, establishment of levels of competency is meaningless outside areas of large Deaf populations.

The use of the Americans with Disabilities Act as an incentive to be more responsive to the deaf population might be severely limited. For many establishments and businesses (especially small organizations, such as solo physicians or small clinics), to become compliant could levy too great a financial hardship or difficulty and thus make these groups exempt.

My office staff reports that sometimes our Deaf patients come to the office already frustrated perhaps in anticipation of problems born of past experiences. There is no question that the Deaf often face considerable problems in obtaining health care.

A final comment on deafness and physicians: most Deaf do not consider their deafness a handi- 
cap; rather, they consider it an inconvenience. The physician's caring and attention will serve to make their deafness less of an inconvenience.

I recall my letter of application to medical school. It ended with the statement, "The Deaf are not an underrepresented minority, we are not represented at all." Today there are more than 40 young hearing-impaired physicians, medical residents, and medical students. Things are changing!

\section{Reference}

1. Witte TN, Kuzel AJ. Elderly deaf patients' health care experiences. J Am Board Fam Pract;2000;13: $17-22$. 